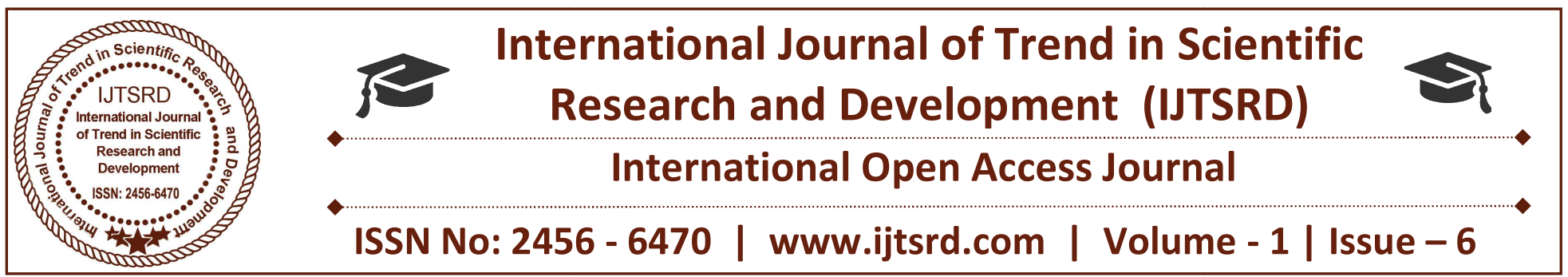

\title{
Phytochemical Profiling and Fluorescence Analysis of Aerial Parts of Vitis vinefera for Modern Health Care Systems
}

\author{
Anita V. Handore \\ Department of Microbiology, HPT Arts and RYK \\ Science College, Nashik, MS, India
}

\author{
Sharad. R. Khandelwal \\ Department of Microbiology, HPT Arts and RYK \\ Science College, Nashik, MS,India
}

\section{ABSTRACT}

In the modern health care system, phytochemical rich plants have widespread applications. Since the ancient times, V.vinefera has been extensively used in various ayurvedic preparations due to presence of different bioactive compounds. However, its phytocontituents shows variation on basis of variety and different parts of plant. The aim of this research is to study the phytochemical profiling and florescence analysis of aerial parts by use of distinct varieties of Vitis vinefera. Healthy aerial parts (leaf lamina, stem and petiole) of different black and white varieties of Vitis vinefera were randomly collected. Organic and aqueous extracts were prepared by soaking the shade dried powder $(10 \%)$ of each part into $70 \%$ ethanol and sterile distill water separately. Phytochemical profiling and fluorescent analysis was carried out by use of reported methods with few modifications .Experimental evaluation showed high abundance of various bioactive phytochemicals in both organic as well as aqueous extracts. It was revealed that organic extracts of both varieties gave best results for some polyphenolic groups like flavonoids, ellagic acid, tannins as well as other groups like glycosides, alkaloids, diterpenes, coumarins, fats and oils,etc. .Only the test for anthocyanin was found to be negative in white variety. It was found that carbohydrates and reducing sugar, proteins and amino acids, saponins as well as tannins has given comparatively good results in aqueous extract. Findings of the fluorescence analysis demonstrated that powdered plant parts treated with different solvents and reagents has shown specific shades of green, brown, pink, black and yellow colour in visible and ultraviolet light. Whereas, some samples showed white, yellow and green fluorescence under short UVlight $(254 \mathrm{~nm})$ and long UV light $(365 \mathrm{~nm})$. Therefore, based on the outcome of this study, it can be concluded that almost all the aerial parts of V.vinefera are rich natural source of various bioactive phytochemicals with clinically proved therapeutic potential. Further investigation for isolation, characterization and purification of such phyto constituents from these cost effective natural sources may become basis for their promising application in the modern health care systems.

Keywords: Vitis vinefera, Chronic diseases Phytochemicals, Fluorescence, Bioactive, Aerial parts, Therapeutic agent

\section{INTRODUCTION}

Ayurveda is the most ancient health care system in India which includes descriptions of various medicinal plants having significant role in modern medicine, not because they continue to be used as crude drug preparations, but because they serve as the 
source of important phytochemicals with important applications in modern therapy. In recent years, developed countries are turning to use traditional medicinal systems as these potent phytochemicals shows defensive mechanism of action against number of chronic diseases. It is reported that phytochemicals like saponins, terpenoids, flavonoids, tannins, steroids and alkaloids have shown significant antiinflammatory activity. Some polycyclic glycosides, tannins, and alkaloids have shown hypoglycemic activities. Terpenoids are known to possess analgesic, immunomodulatory, anti-cancer, anti-inflammatory, anti-viral and antimicrobial properties while, steroids help in reducing cholesterol levels and regulates the immune response [1-6].Proteins and carbohydrates are the building blocks of life, our body needs proteins for repairing and maintaining itself. Whereas, group of coumarins have been increasingly attracting special interest as phytochemicals due to the underlying contributions in prevention and treatment of different diseases viz. anti-inflammatory, anticoagulant, antibacterial, antifungal, antiviral, anticancer, antihypertensive, anti-tubercular, anticonvulsant, antiadipogenic [7-8]. Cardiac glycosides, are also proved as inhibitors of the plasma membrane $\mathrm{Na}+/ \mathrm{K}+-$ ATPase and clinically used for the treatment of heart failure. Some studies have suggested that cardiac glycosides target cancer cells selectively and efficiently [9].

Among all the phytochemicals, polyphenols are clinically proved group of promising phytoconstituent associated with reduced risk of various chronic diseases, including cancer, cardiovascular disease and neurodegenerative disorders. They are present in various plants in the form of different compounds like stilbene (Resveratrol), flavonoid, tannin, ellagic acid, flavonoids etc. of which, resveratrol has emerged as a potent molecule due to its outstanding biological properties. It is reported that plants containing such phytochemicals have been effectively used for more than 2000 years in the traditional medicinal preparations like Drakshasava made from Vitis vinifera. Resveratrol acts as anticancer, cardio protective, anti -diabetic, antioxidant, anti-aging (Life extension), anti-inflammatory , antimicrobial and neuroprotective activity [10]. In $V$.vinefera some type of flavonoids like quercetin can shows antioxidant, anti-allergic, antiinflammatory, antiviral, anti-proliferative, and anticarcinogenic activities, in addition to positive impact on mammalian metabolism [11]. Ellagic acid has a variety of benefits for their anti-mutagenic, antimicrobial and antioxidant properties, and inhibitors of human immunodeficiency virus (HIV). It plays important prevention role in formation of various tumors [12].These phyto-constituents shows characteristic fluorescence property in visible as well as in the UV light which is one of the important parameter of pharmacognostical evaluation. It is stated that when physical and chemical methods are inadequate, the plant material can be identified from their adulterants on the basis of fluorescence analysis.

In V.vinefera, phytochemicals have been proved as therapeutic agents. Their therapeutic efficacy against treatment of different diseases and disorders may vary with individual phytochemical, group of phytochemicals working together or phytochemicals working with other substances. Although V.vinefera contains variety of phytochemicals, its composition varies greatly among different varieties. Therefore, aim of this research is to study the phytochemical profiling and florescence analysis of aerial parts by use of distinct varieties of Vitis vinefera for their promising application in the modern health care systems.

\section{MATERIALS AND METHODS}

\section{Plant Material Collection and Extraction}

Healthy aerial plant parts (leaf -lamina, Stem and petiole) of different black and white varieties of Vitis vinefera viz. Ganesh -white, Sonaka -black, Jumbo black, Sharad Seedless- black were randomly collected from the vineyard of Nashik region, Maharashtra India during June 2016.Collected plant parts were cleaned, cut into small pieces and shade dried at room temperature for about fifteen days and grounded to fine powder for extraction. Organic and aqueous extracts were prepared by soaking the dry powder $(10 \%)$ of each part into $70 \%$ Ethanol and sterile distill water respectively. Incubation was carried out at room temperature with gentle shaking for $72 \mathrm{~h}$.The supernatants obtained were used for further analysis.

\section{Phytochemical Profiling}

Identification of various bioactive phyto-constituents in organic and aqueous plant extract was carried out by use of reported methods with few modifications. Results were identified by visual observation of 
colour change or by precipitate formation on addition of specific reagents to the test solution. [Table.1]

\section{Florescence analysis}

Dried powder samples $(0.5 \mathrm{gm})$ of different aerial plant parts of V.vinefera were taken into clean and dried test tubes. To each tube $5 \mathrm{ml}$ of different organic solvents like glacial acetic acid, acetone, benzene, chloroform, Dil. Hydrochloric acid, Ethanol, 5\% $\mathrm{FeCl}$,Conc. Hydrochloric acid ,Methanol, $1 \mathrm{~N} \mathrm{NaOH}$ + Methanol, $1 \mathrm{~N} \mathrm{NaOH}$,Nitric acid, Petroleum ether, Picric acid, Conc.Sulphuric acid were added separately. Then, the tubes were shaken and allowed to stand for about $30 \mathrm{~min}$. The supernatants were observed in visible light, short UV- light $(254 \mathrm{~nm})$ and long UV light $(365 \mathrm{~nm})$ for their characteristic colour reaction [13]. Colours were recorded on basis of standard colour chart [Table.2]

\section{RESULTS AND DISCUSSION}

Phytochemicals play significant role in defense mechanism by protecting the plant from pathogenic attack, insects, ultraviolet radiations and other environmental stresses. It is clinically proved that phytochemicals has ability to reduce the risk of different chronic diseases and disorders by their ability to neutralize the free radicals responsible for the onset of these diseases.

During the phytochemical profiling, It was found that both organic as well as aqueous extracts showed presence of various bioactive phyto-constituents such as polyphenolic compounds, flavonoids, anthocyanins, carbohydrate, fats and oils, cardiac glycosides, saponin (Fig.1), tannin, alkaloids, ellagic acid ,phytosterols, diterpenes,proteins and amino acids, coumarins etc. in high concentrations .

It was revealed that organic extract showed best results for some groups of polyphenols like flavonoids, anthocyanin, ellagic acid as well as other groups like glycosides, alkaloids, diterpenes, fats and oils, coumarins etc. Whereas, test for anthocyanin was negative in white variety. It was found that aqueous extract showed comparatively best results for carbohydrates and reducing sugar, proteins and amino acids, saponins and tannins .Among all the detected compounds, some test for flavonoids, carbohydrates alkaloids, coumarins, protein and acids and tannins showed satisfactory results in both organic as well as aqueous extract. (Table 1)

Table 1: Phytochemical Screening Tests

\begin{tabular}{|c|c|c|c|c|c|}
\hline $\begin{array}{l}\text { Sr. } \\
\text { No. }\end{array}$ & Group & Test & Reaction & Observation & Reference \\
\hline 1 & Phenols & Ferric chloride & $\begin{array}{l}0.5 \mathrm{ml} \text { extract }+0.5 \mathrm{ml} \\
\text { Ferric chloride }(10 \%)\end{array}$ & $\begin{array}{l}\text { Formation of green } \\
\text { precipitate. }\end{array}$ & [14] \\
\hline \multirow[t]{5}{*}{2} & \multirow[t]{5}{*}{ Flavonoids } & 1. Lead acetate & $\begin{array}{l}0.5 \mathrm{ml} \text { extract }+0.5 \mathrm{ml} \\
\text { lead acetate }(10 \%)\end{array}$ & $\begin{array}{l}\text { Formation of buff } \\
\text { coloured solution (pale } \\
\text { yellow colour). }\end{array}$ & [15] [17] \\
\hline & & 2. $\mathrm{NaOH}$ & $\begin{array}{l}0.5 \mathrm{ml} \text { extract }+0.5 \mathrm{ml} \\
\mathrm{NaOH},(10 \%)+0.5 \mathrm{ml} \\
\text { dil } \mathrm{HCl}\end{array}$ & $\begin{array}{l}\text { Yellow solution turned } \\
\text { colourless on addition } \\
\text { of dil } \mathrm{HCl} \text {. }\end{array}$ & [16-17] \\
\hline & & 3. Shinoda & $\begin{array}{l}\text { Mg chip }(0.5 \mathrm{~cm})+1 \\
\mathrm{ml} \text { extract }+2-3 / \text { few } \\
\text { drops of conc. } \mathrm{HCl}\end{array}$ & $\begin{array}{l}\text { Dark brown solution } \\
\text { turns red with } \\
\text { effervescence. }\end{array}$ & {$[17-18]$} \\
\hline & & $\begin{array}{l}\text { 4.Aluminum } \\
\text { Chloride }\end{array}$ & $\begin{array}{l}1 \mathrm{ml} \text { extract }+0.25 \mathrm{ml} \\
\text { aluminum chloride } \\
\text { solution }(1 \%) \text {, Gentle } \\
\text { shaking }\end{array}$ & $\begin{array}{l}\text { Yellow coloration with } \\
\text { precipitate }\end{array}$ & [17] [19] \\
\hline & & $\begin{array}{l}\text { 5.Sulphuric } \\
\text { acid } \\
\text { (Anthocyanin) }\end{array}$ & $\begin{array}{l}1.5 \mathrm{ml} \text { of extract }+\mathrm{few} \\
\text { drops of } \mathrm{H}_{2} \mathrm{SO}_{4}\end{array}$ & $\begin{array}{l}\text { Development of } \\
\text { yellowish orange } \\
\text { colour. }\end{array}$ & {$[17][20]$} \\
\hline
\end{tabular}


International Journal of Trend in Scientific Research and Development (IJTSRD) ISSN: 2456-6470

\begin{tabular}{|c|c|c|c|c|c|}
\hline \multirow[t]{3}{*}{3} & \multirow[t]{3}{*}{ Carbohydrate } & $\begin{array}{l}\text { 1.Molisch's } \\
\text { solution }\end{array}$ & $\begin{array}{l}1 \mathrm{ml} \text { of Molisch's } \\
\text { solution }+1 \mathrm{ml} \text { extract } \\
+ \text { Slowly addition of } \\
\text { conc. } \mathrm{H}_{2} \mathrm{SO}_{4} \text { along the } \\
\text { side of test tube. }\end{array}$ & $\begin{array}{l}\text { Appearance of violet } \\
\text { ring at the inter phase } \\
\text { of the test tube. }\end{array}$ & {$[15]$} \\
\hline & & $\begin{array}{l}\text { 2.Benedict's } \\
\text { reagent }\end{array}$ & $\begin{array}{l}0.5 \mathrm{ml} \text { of Benedict's } \\
\text { reagent }+0.5 \mathrm{ml} \\
\text { extract ,Boil the } \\
\text { mixure }\end{array}$ & $\begin{array}{l}\text { Formation of reddish } \\
\text { brown colour. }\end{array}$ & [21] \\
\hline & & $\begin{array}{l}\text { 3.Reducing } \\
\text { sugar (DNSA) }\end{array}$ & $\begin{array}{l}1 \mathrm{ml} \text { extract }+1 \mathrm{ml} \\
\text { DNSA,Boil the } \\
\text { mixure }\end{array}$ & $\begin{array}{l}\text { Formation of brown } \\
\text { colour. }\end{array}$ & {$[22]$} \\
\hline 4 & $\begin{array}{l}\text { Cardiac } \\
\text { glycoside }\end{array}$ & Keller-Killiani & $\begin{array}{l}2 \mathrm{ml} \text { extract }+1.5 \mathrm{ml} \\
\mathrm{FeCl}_{3}(3.5 \%)+1 \mathrm{ml} \\
\text { Glacial acetic acid }+ \\
\text { Slowly addition of } \\
\text { conc. } \mathrm{H}_{2} \mathrm{SO}_{4} \text { along the } \\
\text { side of test tube. }\end{array}$ & $\begin{array}{l}\text { Formation of reddish } \\
\text { brown ring at the } \\
\text { interphase of tube test }\end{array}$ & [23] \\
\hline 5 & Saponin & Hemolysis & $\begin{array}{l}100 \mu \mathrm{l} \text { RBC solution } \\
(5 \%)+100 \mu 1 \text { extract, } \\
\text { Incubation at } 37^{\circ} \mathrm{C}, 30 \\
\text { Min ,centrifuge } \\
5000 \mathrm{rpm} \text { for } 5 \mathrm{~min}\end{array}$ & Hemolysis observed & {$[24]$} \\
\hline \multirow[t]{2}{*}{6} & \multirow[t]{2}{*}{ Tannin } & Gelatin & $\begin{array}{l}0.5 \mathrm{ml} \text { extract }+1 \mathrm{ml} \\
\text { gelatin solution }(1 \%)\end{array}$ & $\begin{array}{l}\text { Formation of white } \\
\text { precipitate. }\end{array}$ & {$[25]$} \\
\hline & & $\mathrm{K}_{2} \mathrm{Cr}_{2} \mathrm{O}_{7}$ & $\begin{array}{l}0.5 \mathrm{ml} \text { extract }+ \text { few } \\
\text { drops of } \mathrm{K}_{2} \mathrm{Cr}_{2} \mathrm{O} 7 \\
\text { solution }(10 \%)\end{array}$ & $\begin{array}{l}\text { Formation of red } \\
\text { precipitate. }\end{array}$ & {$[18]$} \\
\hline 7 & Alkaloids & $\begin{array}{l}\text { Mayer's } \\
\text { reagent }\end{array}$ & $\begin{array}{l}1 \mathrm{ml} \text { Extract }+1 \mathrm{ml} \\
\text { Mayer's reagent }\end{array}$ & $\begin{array}{l}\text { Formation of cream } \\
\text { colour precipitate. }\end{array}$ & {$[20]$} \\
\hline 8 & Ellagic acid & Ellagic acid & $\begin{array}{l}1 \mathrm{ml} \text { Extract }+ \text { few } \\
\text { drops of glacial acetic } \\
\text { acid }(5 \%)(\mathrm{w} / \mathrm{v}) \text { and }+ \\
\text { few drops of } \\
\mathrm{NaNO}_{2} \text { solution }(5 \% \\
\mathrm{w} / \mathrm{v})\end{array}$ & $\begin{array}{l}\text { Formation of Muddy } \\
\text { or Niger brown } \\
\text { precipitate. }\end{array}$ & [23] \\
\hline 9 & Phytosterols & Salkowski’s & $\begin{array}{l}1 \mathrm{ml} \text { extract }+1 \mathrm{ml} \\
\mathrm{CHCl}_{3}+\mathrm{Few} \text { drops } \\
\text { Conc. } \mathrm{H}_{2} \mathrm{SO}_{4}\end{array}$ & $\begin{array}{l}\text { Formation of reddish } \\
\text { brown colour at } \\
\text { interphase of test tube. }\end{array}$ & {$[15]$} \\
\hline 10 & Diterpenes & Copper acetate & $\begin{array}{l}1 \mathrm{ml} \text { Extracts }+3-4 \\
\text { drops of copper } \\
\text { acetate solution }(2 \%)\end{array}$ & $\begin{array}{l}\text { Formation of emerald } \\
\text { green colour. }\end{array}$ & {$[26]$} \\
\hline \multirow[t]{2}{*}{11} & \multirow[t]{2}{*}{$\begin{array}{l}\text { Proteins and } \\
\text { amino acids }\end{array}$} & 1.Xanthoproteic & $\begin{array}{l}1 \mathrm{ml} \text { extracts }+ \text { few } \\
\text { drops of Conc. Nitric } \\
\text { acid }\end{array}$ & $\begin{array}{l}\text { Formation of Yellow } \\
\text { colour. } \\
\text {. }\end{array}$ & {$[18]$} \\
\hline & & 2. Ninhydrin & $\begin{array}{l}1 \mathrm{ml} \text { extract }+1 \mathrm{ml} \\
\text { Ninhydrin reagent } \\
(0.25 \%), \text { Boiled for } \\
\text { few minutes }\end{array}$ & $\begin{array}{l}\text { Formation of blue } \\
\text { colour. }\end{array}$ & {$[27]$} \\
\hline 12 & Fats and oils & Saponification & $\begin{array}{l}\text { 1ml Extract+ few } \\
\text { drops alcoholic } \mathrm{KOH}\end{array}$ & $\begin{array}{l}\text { Formation of soap or } \\
\text { partial neutralization }\end{array}$ & [28] \\
\hline
\end{tabular}


International Journal of Trend in Scientific Research and Development (IJTSRD) ISSN: 2456-6470

\begin{tabular}{|l|l|l|l|l|l|}
\hline & & $\begin{array}{l}(0.5 \mathrm{~N})+\text { few drops of } \\
\text { Phenolphthalein } \\
\text {,heating on a water } \\
\text { bath for } 1-2 \mathrm{~h}\end{array}$ & of alkali & \\
\hline $\mathbf{1 3}$ & Coumarins & $\mathrm{NaOH}$ & $\begin{array}{l}1 \mathrm{ml} \text { extract }+1.5 \mathrm{ml} \\
\text { NaOH }(10 \%)\end{array}$ & $\begin{array}{l}\text { Formation of yellow } \\
\text { colour. }\end{array}$ & {$[16]$} \\
\hline
\end{tabular}

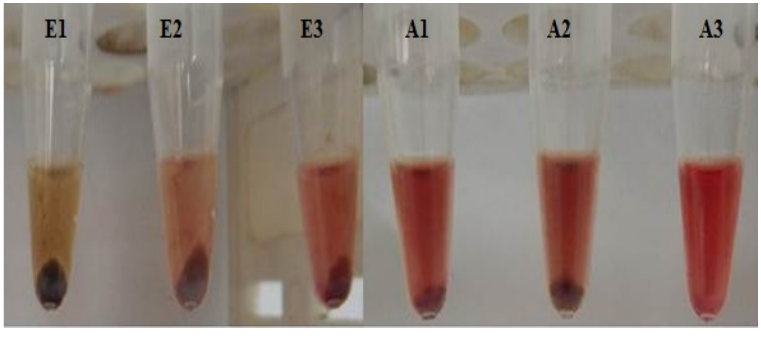

[1. Ganesh White]

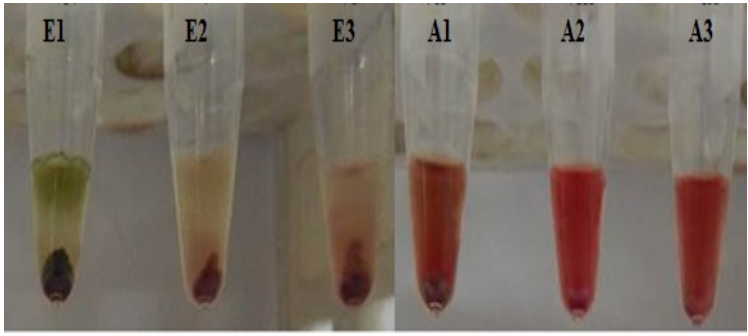

[3. Sonaka -Black ]

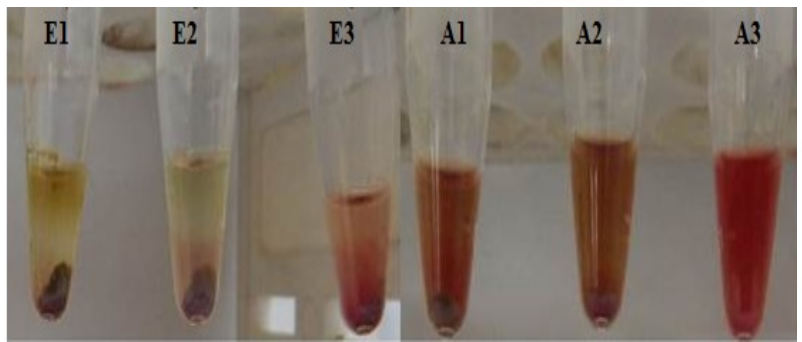

[2. Jumbo-Black]

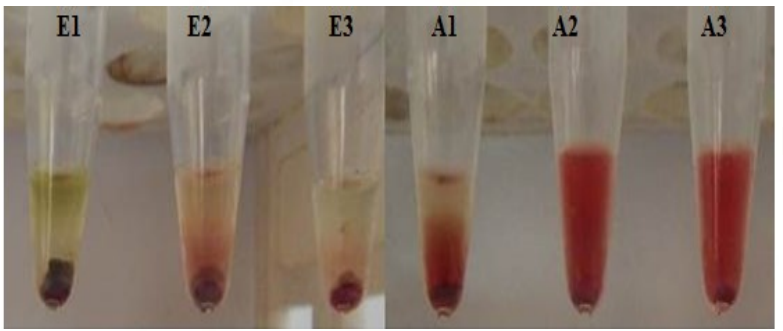

[4. Sharad seedless -Black]

[E1.Ethanolic Extract -Leaf lamina, E2.Ethanolic Extract -Stem, E3. Ethanolic Extract -Petiole A1.Aqueous Extract -Leaf lamina, A2. Aqueous Extract -Stem, A3. Aqueous Extract -Petiole]

\section{Fig.1. Hemolysis Test for Saponin by Ethanolic and Aqueous Extract of Different Aerial Parts of V.vinefera}

\section{Fluorescence Analysis}

Fluorescence property is exhibited by various chemical constituents present in the plant material. Ultraviolet light produces fluorescence in many natural products which do not shows fluoresce in visible light. Although, some substance are not fluorescent, they may be often converted into fluorescent derivatives by use of different chemical reagents and solvents.Fluorescence is the most important parameter of pharmacognostical evaluation so,qualitative analysis of some crude drugs using fluorescence is widely carried out [29]. There is a correlation between a compound present in the drugs and their fluorescent behavior at different wavelengths due to the presence of different chemical constituents in the drug. [30].During the Fluorescence analysis, it was observed that the powdered plant parts of V.vinefera showed different shades of green brown, pink and yellow colours in visible and Ultraviolet light, whereas some of the samples gives white, yellow and green fluorescence under short UVlight (254 $\mathrm{nm})$ and long UV light (365 nm) after treatment with different solvents [Table. 2 and Fig.2.] 
International Journal of Trend in Scientific Research and Development (IJTSRD) ISSN: 2456-6470

Table.2.Fluorescent analysis of aerial parts of $V$.vinefera in different chemical reagents and solvents under different wavelengths

\begin{tabular}{|c|c|c|c|c|c|c|c|c|c|}
\hline \multirow{2}{*}{$\begin{array}{l}\text { Organic } \\
\text { Solvents }\end{array}$} & \multicolumn{3}{|c|}{ Visible Light } & \multicolumn{3}{|c|}{$\begin{array}{l}\text { Under long UV } \\
(\lambda \max 365 \mathrm{~nm})\end{array}$} & \multicolumn{3}{|c|}{$\begin{array}{l}\text { Under Short UV } \\
(\lambda \max 254 \text { nm) }\end{array}$} \\
\hline & $\begin{array}{l}\text { Leaf } \\
\text { Lamina }\end{array}$ & Stem & Petiole & $\begin{array}{c}\text { Leaf } \\
\text { Lamina }\end{array}$ & Stem & Petiole & $\begin{array}{c}\text { Leaf } \\
\text { Lamina }\end{array}$ & Stem & Petiole \\
\hline $\begin{array}{l}\text { Glacial } \\
\text { acetic } \\
\text { acid }\end{array}$ & $\begin{array}{l}\text { Deep } \\
\text { Olive } \\
\text { Green }\end{array}$ & Brown & $\begin{array}{l}\text { Light } \\
\text { Brown }\end{array}$ & Deep Pink & $\begin{array}{l}\text { Light } \\
\text { Pink }\end{array}$ & $\begin{array}{l}\text { Light } \\
\text { Pink }\end{array}$ & $\begin{array}{l}\text { Deep } \\
\text { Olive } \\
\text { Green }\end{array}$ & Yellow & $\begin{array}{l}\text { Greeni } \\
\text { sh } \\
\text { yellow }\end{array}$ \\
\hline Acetone & $\begin{array}{l}\text { Light } \\
\text { Olive } \\
\text { Green }\end{array}$ & $\begin{array}{l}\text { Light } \\
\text { yellow }\end{array}$ & $\begin{array}{l}\text { Light } \\
\text { yellow }\end{array}$ & Pink & $\begin{array}{l}\text { Light } \\
\text { Pink }\end{array}$ & $\begin{array}{l}\text { Light } \\
\text { Pink }\end{array}$ & $\begin{array}{l}\text { Olive } \\
\text { Green }\end{array}$ & $\begin{array}{l}\text { Light } \\
\text { yellow }\end{array}$ & $\begin{array}{l}\text { Light } \\
\text { yellow }\end{array}$ \\
\hline Benzene & $\begin{array}{l}\text { Brownish } \\
\text { Green }\end{array}$ & $\begin{array}{l}\text { Light } \\
\text { Green }\end{array}$ & light Green & Deep Pink & Pink & Pink & $\begin{array}{l}\text { Olive } \\
\text { Green }\end{array}$ & $\begin{array}{l}\text { Yellow } \\
\text { ish } \\
\text { green }\end{array}$ & $\begin{array}{l}\text { Yellow } \\
\text { ish } \\
\text { green }\end{array}$ \\
\hline $\begin{array}{l}\text { Chlorof } \\
\text { orm }\end{array}$ & $\begin{array}{l}\text { Brownish } \\
\text { green }\end{array}$ & $\begin{array}{l}\text { Light } \\
\text { Brown }\end{array}$ & Colourless & Deep Pink & Pink & Pink & $\begin{array}{l}\text { Olive } \\
\text { Green }\end{array}$ & $\begin{array}{l}\text { Yellow } \\
\text { ish } \\
\text { green }\end{array}$ & $\begin{array}{l}\text { Yellow } \\
\text { ish } \\
\text { green }\end{array}$ \\
\hline Dil. HCl & $\begin{array}{l}\text { Brownish } \\
\text { yellow }\end{array}$ & $\begin{array}{l}\text { Light } \\
\text { Brown }\end{array}$ & $\begin{array}{l}\text { Light } \\
\text { Brown }\end{array}$ & Pink & Light Pink & Light Pink & $\begin{array}{l}\text { Fluorescen } \\
\text { ce Green }\end{array}$ & $\begin{array}{l}\text { Light } \\
\text { yellow }\end{array}$ & $\begin{array}{l}\text { Light } \\
\text { yellow }\end{array}$ \\
\hline Ethanol & $\begin{array}{l}\text { Deep } \\
\text { Olive } \\
\text { Green }\end{array}$ & $\begin{array}{l}\text { Light Olive } \\
\text { Green }\end{array}$ & $\begin{array}{l}\text { Light Olive } \\
\text { Green }\end{array}$ & Deep Pink & Light Pink & Light Pink & $\begin{array}{l}\text { Deep Olive } \\
\text { Green }\end{array}$ & $\begin{array}{l}\text { Fluoros } \\
\text { cent } \\
\text { yellow }\end{array}$ & $\begin{array}{l}\text { Fluoros } \\
\text { cent } \\
\text { yellow }\end{array}$ \\
\hline $\begin{array}{l}5 \% \\
\text { Ferric } \\
\text { chloride }\end{array}$ & $\begin{array}{l}\text { Deep } \\
\text { Brownish } \\
\text { green }\end{array}$ & $\begin{array}{l}\text { Greenish } \\
\text { orange }\end{array}$ & Orange & Deep Pink & Light Pink & Light Pink & $\begin{array}{l}\text { Deep Olive } \\
\text { Green }\end{array}$ & $\begin{array}{l}\text { Deep } \\
\text { Olive } \\
\text { Green }\end{array}$ & $\begin{array}{l}\text { Light } \\
\text { Olive } \\
\text { Green }\end{array}$ \\
\hline $\begin{array}{l}\text { Conc. } \\
\mathrm{HCl}\end{array}$ & $\begin{array}{l}\text { Blackish } \\
\text { Green }\end{array}$ & $\begin{array}{l}\text { Muddy } \\
\text { brown }\end{array}$ & $\begin{array}{l}\text { Muddy } \\
\text { brown }\end{array}$ & Pink & Light Pink & Light Pink & $\begin{array}{l}\text { Olive } \\
\text { Green }\end{array}$ & $\begin{array}{l}\text { Greeni } \\
\text { sh } \\
\text { yellow }\end{array}$ & $\begin{array}{l}\text { Greeni } \\
\text { sh } \\
\text { yellow }\end{array}$ \\
\hline $\begin{array}{l}\text { Methan } \\
\text { ol }\end{array}$ & $\begin{array}{l}\text { Deep } \\
\text { Olive } \\
\text { Green }\end{array}$ & $\begin{array}{l}\text { Light } \\
\text { Green }\end{array}$ & Light Green & Deep Pink & Colourless & Colourless & $\begin{array}{l}\text { Olive } \\
\text { Green }\end{array}$ & $\begin{array}{l}\text { Yellow } \\
\text { ish } \\
\text { green }\end{array}$ & $\begin{array}{l}\text { Yellow } \\
\text { ish } \\
\text { green }\end{array}$ \\
\hline $\begin{array}{l}1 \mathrm{~N} \\
\mathrm{NaOH} \\
+ \text { Metha } \\
\text { nol }\end{array}$ & $\begin{array}{l}\text { Deep } \\
\text { Olive } \\
\text { Green }\end{array}$ & $\begin{array}{l}\text { Brownish } \\
\text { orange }\end{array}$ & Light brown & Deep Pink & Colourless & Colourless & $\begin{array}{l}\text { Deep Olive } \\
\text { Green }\end{array}$ & $\begin{array}{l}\text { Deep } \\
\text { Olive } \\
\text { Green }\end{array}$ & $\begin{array}{l}\text { Light } \\
\text { Olive } \\
\text { Green }\end{array}$ \\
\hline $\begin{array}{l}1 \mathrm{~N} \\
\mathrm{NaOH}\end{array}$ & $\begin{array}{l}\text { Deep } \\
\text { reddish } \\
\text { orange }\end{array}$ & $\begin{array}{l}\text { Reddish } \\
\text { orange }\end{array}$ & $\begin{array}{l}\text { Light } \\
\text { orange }\end{array}$ & Deep Pink & Colourless & Colourless & $\begin{array}{l}\text { Deep Olive } \\
\text { Green }\end{array}$ & $\begin{array}{l}\text { Light } \\
\text { Olive } \\
\text { Green }\end{array}$ & $\begin{array}{l}\text { Light } \\
\text { Olive } \\
\text { Green }\end{array}$ \\
\hline $\begin{array}{l}\text { Nitric } \\
\text { Acid }\end{array}$ & $\begin{array}{l}\text { Brownish } \\
\text { yellow }\end{array}$ & $\begin{array}{l}\text { Light } \\
\text { brownish } \\
\text { yellow }\end{array}$ & $\begin{array}{l}\text { Light } \\
\text { brownish } \\
\text { yellow }\end{array}$ & $\begin{array}{l}\text { Fluoroscent } \\
\text { white }\end{array}$ & $\begin{array}{l}\text { Fluoroscent } \\
\text { white }\end{array}$ & $\begin{array}{l}\text { Fluoroscent } \\
\text { white }\end{array}$ & $\begin{array}{l}\text { Fluoroscent } \\
\text { Green }\end{array}$ & $\begin{array}{l}\text { Fluoros } \\
\text { cent } \\
\text { Yellow }\end{array}$ & $\begin{array}{l}\text { Fluoros } \\
\text { cent } \\
\text { Yellow }\end{array}$ \\
\hline $\begin{array}{l}\text { Petroleu } \\
\text { m Ether }\end{array}$ & $\begin{array}{l}\text { Brownish } \\
\text { green }\end{array}$ & Colourless & Colourless & Pink & Light Pink & Light Pink & $\begin{array}{l}\text { Fluoroscent } \\
\text { Green }\end{array}$ & $\begin{array}{l}\text { Colourl } \\
\text { ess }\end{array}$ & $\begin{array}{l}\text { Colourl } \\
\text { ess }\end{array}$ \\
\hline $\begin{array}{l}\text { Picric } \\
\text { Acid }\end{array}$ & $\begin{array}{l}\text { Greenish } \\
\text { orange }\end{array}$ & $\begin{array}{l}\text { Light } \\
\text { orange }\end{array}$ & $\begin{array}{l}\text { Light } \\
\text { Orange }\end{array}$ & $\begin{array}{l}\text { Blackish } \\
\text { pink }\end{array}$ & Deep pink & Deep pink & $\begin{array}{l}\text { Fluoroscent } \\
\text { Green }\end{array}$ & $\begin{array}{l}\text { Fluoros } \\
\text { cent } \\
\text { Green }\end{array}$ & $\begin{array}{l}\text { Fluoros } \\
\text { cent } \\
\text { Green }\end{array}$ \\
\hline $\begin{array}{l}\text { Conc. } \\
\mathrm{H}_{2} \mathrm{SO}_{4}\end{array}$ & $\begin{array}{l}\text { Greenish } \\
\text { Brown }\end{array}$ & $\begin{array}{l}\text { Reddish } \\
\text { Brown }\end{array}$ & $\begin{array}{l}\text { Reddish } \\
\text { Brown }\end{array}$ & $\begin{array}{l}\text { Blackish } \\
\text { Pink }\end{array}$ & $\begin{array}{l}\text { Blackish } \\
\text { Pink }\end{array}$ & $\begin{array}{l}\text { Blackish } \\
\text { Pink }\end{array}$ & $\begin{array}{l}\text { Blackish } \\
\text { Green }\end{array}$ & $\begin{array}{l}\text { Blackis } \\
\mathrm{h} \\
\text { Green }\end{array}$ & $\begin{array}{l}\text { Blackis } \\
\mathrm{h} \\
\text { Green }\end{array}$ \\
\hline
\end{tabular}



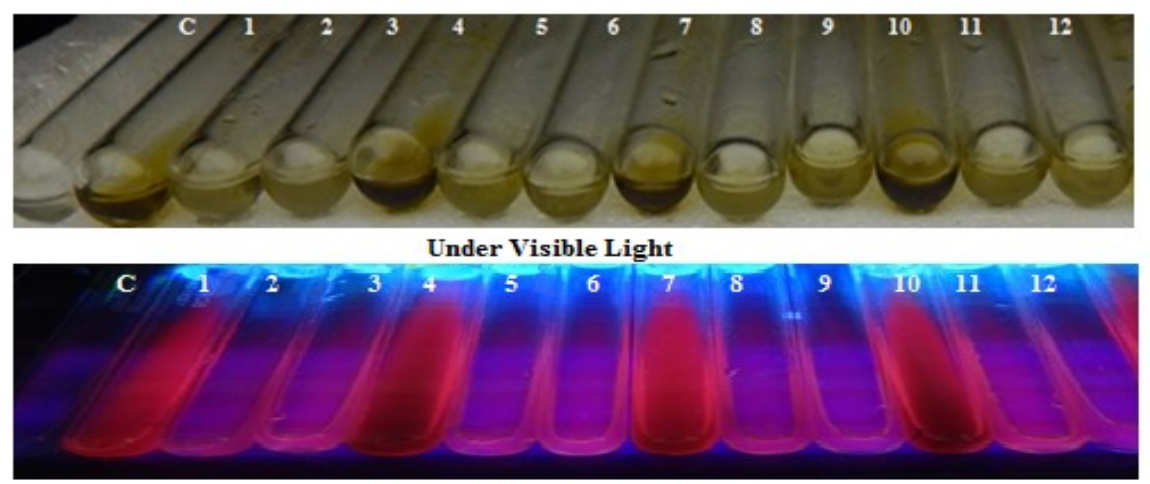

Under long $\mathrm{UV}, \lambda \max =365 \mathrm{~nm}$

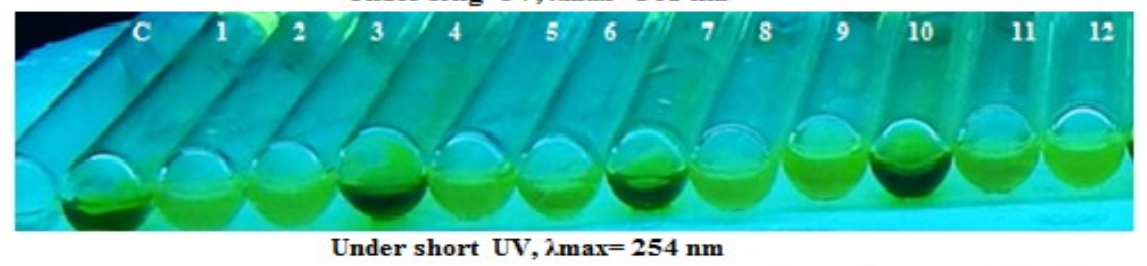

[Fig.2a. Solvent : Benzene]

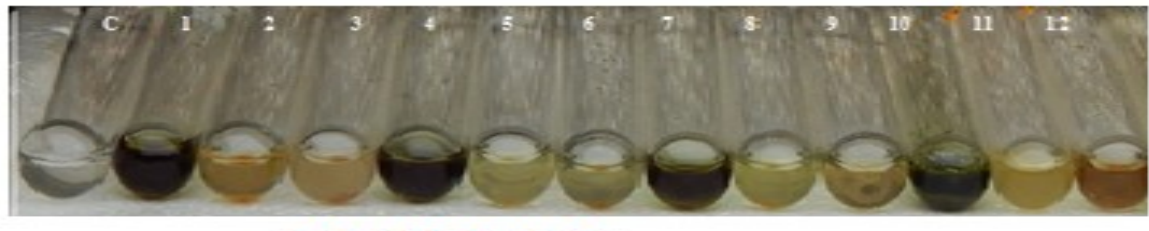

Under Visible light

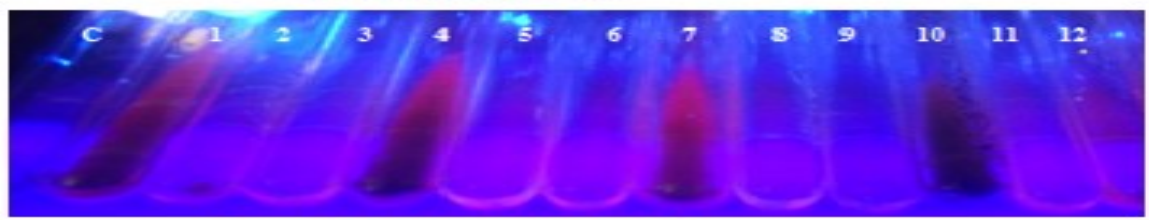

Under Long UV, $\lambda$ max $=365 \mathrm{~nm}$

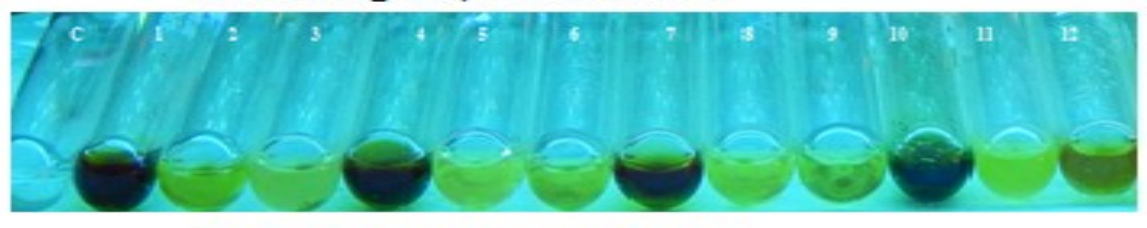

Under short UV, 2max $=254 \mathrm{~mm}$

[Fig.2b. Solvent: Glacial Acetic acid]

C.Negative control, 1.Gaesh -Leaf lamina , 2. Gaesh-Stem, 3.Gaesh-Petiole 4. Jumbo Leaf lamina 5. Jumbo Stem 6. Jumbo Petiole 7.Sonaka - Leaf lamina 8.Sonaka - Stem 9.Sonaka- Petiole 10.Sharad Seedless- Leaf lamina , 11. Sharad Seedless- Stem, 12.Sharad Seedless- Petiole

Fig.2. Fluorescent Analysis of aerial parts of different varieties of $V$.vinefera.

\section{CONCLUSION}

In the present study, almost all the aerial parts of both varieties of $V$.vinefera showed presence of various phyto-constituents having clinically proved promising role against number of chronic diseases. Results obtained during phytochemical profiling indicate that

both the organic as well as aqueous extracts showed high abundance of various bioactive phytochemicals.
It was revealed that organic solvents showed best results for some polyphenolic groups like flavonoids, anthocyanin, ellagic acid as well as other groups like glycosides, alkaloids, diterpenes, coumarins, fats and oils, etc. whereas, test for anthocyanin was negative in white variety It was found that aqueous extract showed comparatively best results for carbohydrates and reducing sugar, proteins and amino acids, saponins and tannins. It was noticed that some detection test for flavonoids, carbohydrates, alkaloids, 
coumarins, protein and acids and tannins gives satisfactory results for both the extracts. Findings of fluorescence analysis demonstrated that some powdered plant parts of V.vinefera treated with different solvents and reagents showed specific colour shades viz. green, brown, pink, black and yellow in visible and ultraviolet light. Whereas, some samples showed white, yellow and green fluorescence under short UV- light (254 nm) and long UV light (365 nm). Therefore, based on the outcome of this study, it can be concluded that aerial parts of V.vinefera are rich natural source of various bioactive phytochemicals with noteworthy health potential. Further investigation for isolation, characterization and purification of such phyto constituents from these cost effective natural sources may become basis for their promising application in the modern health care systems.

\section{ACKNOWLEDGEMENT}

The authors are grateful to Prin. V. N. Suryavanshi and Dr. L.P.Sharma, HOD, Department of Microbiology, H.P.T .Arts and R.Y.K. Science College, Nashik, India for providing the necessary facilities. We acknowledge J.W.Baviskar and for the assistance. Authors are highly thankful to Mr. D.V.Handore, Research Mentor, Sigma Winery Pvt. Ltd. Nashik for valuable scientific inputs.

\section{REFERENCES}

1) H.P Rupasinghe, C.J Jackson, V Poysa, J.Di Berado,

J.D.

Bewley, J.Jenkinson., "Soyasapogenol A and B distribution in Soyabean (Glycine max (1.) Merr.) in relation to seed physiology ,genetic variability and growing location", Journal of Agricultural and Food Chemistry 51, 2003. pp.5888-5894

2) W Salah, NJ Miller, G Pagauga, Tijburg, GP Bolwell, E Rice, C Evans, "Polyphenolic flavonols as scavenger of aqueous phase radicals and chainbreaking antioxidants",Arch. Biochem. Biol. 2, 1995,pp.339- 346

3) A. Rio Del ,B G. Obdululio, J.Casfillo, F.G. Main, A.Ortuno, "Uses and properties of citrus flavonoids", Afr. J. Biotechnol,4(7), 1997, pp.685688.

4) K.H Wagner, I Elmadfa, "'Biological relevance of terpenoids: Overview focusing on mono-di and tetraterpenes", Ann. Nutr. Metab, 47, 2003, pp.95106.
5) B. F Sultana, M.R Anwar, S. Asi.A.S. Chatha, Antioxidant potential of extracts from different agro wastes: Stabilization of corn oil. Grasas Y Aceites, 59,2008,pp.205-217

6) Rabi , Bishayee A, "Terpenoids and breast cancer chemoprevention. Breast Cancer Res. Treat.", 115,2009,pp.223-239

7) K.N Venugopala, V.Rashmi, B. Odhav, "Review on Natural Coumarin Lead Com- pounds for Their Pharmacological Activity",BioMed Research International, 2013,pp.1-14.

8) M.E Riveiro, N .De Kimpe, A. Moglioni, R .Vazquez, F. Monczor, C. Shayo, "Coumarins: Old Compounds with Novel Promising Therapeutic Perspectives",Curr Med Chem - AntiCancer Agents, 17(13), 2010,pp.1325-38.

9) M López-Lázaro, "Digi toxin as an anticancer agent with selectivity for cancer cells: possible mechanisms involved",Expert Opinion on Therapeutic Targets, 11,2007,pp.1043-1053

10) A.V. Handore and S. R .Khandelwal,"Resveratrol -The Nutraceutical, Whose Real Time Has Come: A Systematic Review", International Journal of Advanced Biotechnology and Research, 8(2), 2017, pp.1516-1544

11) W. Ren,Z, Oiao, H. Wang ,L.Zhu and L.Zhang, Flavonoids;Promising anticancer agents Med.Res.Rev.,23,2003,pp.519-534

12) L. Sepúlveda, A. Ascacio , R.Rodríguez-Herrera, A. Aguilera-Carbó and $\mathrm{N}$ Cristóbal.Aguila, , "Ellagic acid:Biological properties and biotechnological development for production processes",African Journal of Biotechnology,10(22), 2011,pp. 4518-4523.

13) R. Kavitha, "Fluorescence and Ft-Ir Analysis of Leaf And Fruit of Trichosanthes Dioica Roxb", World Journal Of Pharmacy And Pharmaceutical Sciences, 3(10), 2014,pp.563-572

14) H Usman, F .I. Abdulrahman, and A Usman, "Qualitative Phytochemical Screening and In Vitro Antimicrobial Effects of Methanol Stem Bark Extract of Ficus Thonningii (Moraceae)", Afr. J.

Trad. CAM.6 (3), 2009, pp.289 - 295

15) S. Mandal, P. Arpita ,A. Samanta, S.Roy, A.Mandal, T.Das Mahapatra, S.Pradhan, K.Das, and D. Kumar Nandi, "Analysis of phytochemical profile of Terminalia arjuna bark extract with 
International Journal of Trend in Scientific Research and Development (IJTSRD) ISSN: 2456-6470

antioxidative and antimicrobial properties",Asian Pac J Trop Biomed, 3(12),2013,pp.960-966

16) M. Majid, Muhammad R. Khan, N. A.Shah, I.U1 Haq, A.F.Muhammad, S.Ullah, A. Sharif, Z. Zahra, T. Younis, and M. Sajid, "Studies on phytochemical, antioxidant,anti-inflammatory and analgesic activities of Euphorbia dracunculoides",BMC Complementary and Alternative Medicine ,2015.pp.15:349

17) A.V. Handore and S.R.Khandelwal, "Screening of Vitis Vinifera for flavonoid content and free radical scavenging potential, International Research Journal of Pharmacy ,2017, 8 (8),pp.1-4

18) S. Sabiha., M .Aftab. Ahmad, M. Asif, A Mohd.and S. Ibne, "Physicochemical and phytochemical Standardization of berries of Myrtus communis Linn", J Pharm Bioallied Sci.,4(4) ,2012,pp.322-

326

19) L. Obasi Nnamdi, C. Egbuonu Anthony. C, O. Ukoha Pius., M Ejikeme Paul,“Comparative phytochemical and antimicrobial screening of some solvent extracts of Samanea saman(fabaceae or mimosaceae) pods", African Journal of Pure and Applied Chemistry , 4(9), 2010,pp. 206-212

20) D. Kalita., N Devi., D. Baishya ,"Comparative Preliminary Foliar Phytochemical Screening of Diospyros malabrica (Desr.) Kostel and Diospyros lanceifolia Roxb", International Journal of Pharmacognosy and Phytochemical Research. 2016; 8(2),pp.239-243

21) C.S.Vimalkumar, V.B Hosagaudar, S.R Suja, V Vilash, N.M Krishnakumar, P.G Latha, "Comparative preliminary phytochemical analysis of ethanolic extracts of leaves of Olea dioica Roxb., infected with the rust fungus Zaghouania oleae (E.J.Butler) Cummins and non-infected plants", Journal of Pharmacognosy and Phytochemistry", 3(4), 2014,pp.69-72

22) V. Alexander, G, Gusakov. E. Kondratyeva, and P Arkady Sinitsyn, "Comparison of Two Methods for Assaying Reducing Sugars in the Determination of Carbohydrase Activities", International Journal of Analytical Chemistry,2011,pp.2-4

23) S.Rimjhim., N. Kumari. K , Jainendra ,Preliminary Phytochemical Screening of
Methanolic Extract of Clerodendron infortunatum",IOSR Journal of Applied Chemistry, 7, 2014,pp.110-13

24) A Elena. Khatuntseva, M. Vladimir Men'shov, S Alexander. Shashkov, E. Yury Tsvetkov, Rodion N. Stepanenko, Ya. Raymonda Vlasenko, E Elvira. Shults, A. Genrikh Tolstikov, G Tatjana. Tolstikova, S Dimitri. Baev, A Vasiliy,A Kaledin, Nelli. Popova, Valeriy P. Nikolin, Pavel P. Laktionov, Anna V. Cherepanova, Tatiana V. Kulakovskaya, Ekaterina V. Kulakovskaya and Nikolay E. Nifantiev, "Triterpenoid saponins from the roots of Acanthophyllum gypsophiloides Regel”, Beilstein J. Org. Chem. 8,2012,pp.763775.

25) S. K. Bhandary, K.N. Suchetha, S. Vadisha Bhat, K.P Sharmila. , M. P.Bekal, "Preliminary Phytochemical Screening Of Various Extracts Of Punica Granatum Peel, Whole Fruit And Seeds", Nitte University Journal of Health Science, 2(4),2012,pp.2249-7110

26) M. Singh, M .Kaur ,C.B.S, Dangi, H Singh "Phytochemical \& TLC Profile of Lawsonia Inermis (Heena)", International Journal for Pharmaceutical Research Scholars , 3(1) ,2014,pp. 624-634

27) G. Iram, M. Sohail, S.A.Muhammad , A.A.Muhammad, "Phytochemical, toxicological and antimicrobial evaluation of lawsonia inermis extracts against clinical isolates of pathogenic bacteria, Annals of Clinical Microbiology and Antimicrobials 2013, 12:36

28) R. Saad et al, "Phytochemical Screening And Antioxidant Activity Of Different Parts From Five Malaysian Herbs", The Experiment, 2014, Vol. 19(2),pp.1336-1347

29) M.K., Gupta, et al., Pharmacognostical evaluation of Grewia asiatica International Journal of Plant Sciences, 2006, 1(2), pp. 249-251.

30) S.H Ansari, Essential of Pharmacognosy, 1st Edition Birla publications Pvt. Ltd, New Delhi 2006. 